Een magazijn-catalogus van J. J. Hummel te Amsterdam en B. Hummel te 's Gravenhage, 1778 Author(s): J. W. Enschedé

Source: Tijdschrift der Vereeniging voor Noord-Nederlands Muziekgeschiedenis, Deel 8, 3de/4de Stuk (1908), pp. 262-286

Published by: Koninklijke Vereniging voor Nederlandse Muziekgeschiedenis

Stable URL: http://www.jstor.org/stable/947983

Accessed: $22 / 06 / 2014$ 10:55

Your use of the JSTOR archive indicates your acceptance of the Terms \& Conditions of Use, available at http://www.jstor.org/page/info/about/policies/terms.jsp

JSTOR is a not-for-profit service that helps scholars, researchers, and students discover, use, and build upon a wide range of content in a trusted digital archive. We use information technology and tools to increase productivity and facilitate new forms of scholarship. For more information about JSTOR, please contact support@jstor.org. 


\section{EEN MAGAZIJN-CATALOGUS VAN J. J. HUMMEL TE AMSTERDAM EN B. HUMMEL TE 'S GRAVENHAGE, 1778.}

Tot de muzikale aantrekkelijkheden van het Amsterdam uit het einde der achttiende eeuw behoorde de inuziekwinkel van Johans Juluus Hummel. die, zijn de verkiegen indrukken daaromtrent juist, niet slechts een aanzienlijke uitgeverszaak dreef, maar ook een goed voorzienen en goed beklanten debiethandel had. $\mathrm{Er}$ is hem aangaande echter heel weinig bekend en geslaagd ben ik er niet in dat weinige te vermeerderen zoodanig, dat van zijn persoonlijkheid en zijn handel ook maar een eenigszins scherp beeld verkregen kan worden. Ik bepaal mij daarom bij elkaar te stellen zonder veel critiek te oefenen, wat elders bericht wordt.

Geboren te Berlijn in 1723, verkrijgt hij 22 Mei 1751 het burgerrecht te 's Gravenhage '). Zijn broeder Burchard verkrijgt aldaar 20 October 1755 hetzelfde recht, terwijl hij zelf 14 Mei 1756 als poorter te Amsterdam wordt ingeschreven. In de acte van inschrijving wordt hij genoemd „musiek winkelier", herkomstic van Watershausen, een mij niet bekende plaats.

Gemeend kan worden dat de beide broeders in Den Haag een muziekhandel in het "Lang Achterom" ') hadden. Er moet dus verondersteld worden, dat in of omstreeks 1755 de haagsche zaak afgetakt is met een amsterdamsche branche, dat ieder der beide broeders aan het hoofd der filialen kwam te staan met dien verstande, dat Johann Julius, de leider der amsterdamsche zaak, zich meer in het bijzonder bezig zou houden met het uitgeven. De haagsche afdeeling, zoowel de oorspronkelijke vóór de verhuizing van Johans Julius naar Amsterdam, als die na

1) D F. Scheurlerk. Mozart's verblijf in Nederland. 's Graven!. 1883, p. З5. 
de splitsing, moet, dunkt me, zich eerst alleen bepaald hebben tot een onderdeeI van den handel, want in 1759 werd Burchard door het gilde aldaar toegelaten tot het verkoopen van muziek en hoogduitsche boeken en eerst in 1762 werd hij als boekverkooper geadmitteerd ${ }^{1}$ ).

De amsterdamsche afdeeling was gevestigd tot 1763 in de Nes, daarna op den Vijgendam ${ }^{1}$ ), later in 1778 was zij te vinden, blijkens achterstaanden magazijn-catalogus, in de Warmoesstraat óver den eersten bijbel.

Johans Julus bleef echter niet in Amsterdam: hij vertrok naar Berlijn en liet aan zijn dochter Euss. Christ. von Metringh en haar echtgenoot het beheer van het amsterdamsche filiaal over. In welk jaar dit geschiedde, weet ik niet; evenmin kan ik verklaren hoe de dochter van hem, Hummer, den eigen geslachtsnaam von Metтingh voert, tenzij dit de familienaam van den schoonzoon is, welke naam echter niet teruggevonden is in de registers van het amsterdamsche gemeente-archief. Ik geef dit bericht dus zoo, als ik het bij Gerber ${ }^{2}$ ) vond, meenende het feit te mogen stellen op 1782, en niet op 1774 wat EitNER doet in zijn Quellen-lexikon op gezag van Forkel.

JoHanN JuLius is nimmer lid geweest van het amsterdamsche boekverkoopersgilde, wat mij administratief gezien zeker vreemd voorkomt. Toch kent de naamlijst van dat gilde een Hummer: in 1782 (13 Mei) werd Leonardus Hummel, de zoon van Burchard uit den Haag, ingeschreven. Er is reden te gelooven, dat deze bij het vertrek van zijn oom naar Berlijn door zijn vader in het amsterdamsch filiaal geplaatst werd. Want terwijl de haagsche firma later werd B. Hummel \& $Z_{N}$., koint diezelfde firmanaam, twee jaar na de inschrijving van LEonardus, eveneens in Amsterdam voor. Het Naamregister van alle de kooplieden der stad Amsterdam voor 1784 - oudere jaargangen van dit adresboek zijn niet meer aanwezig - drukt op blz. 50:

Hummel en Zoon, (Burgh.) in de Warmoesstr. naast de 1ste Liesveldsche Bybel, in Musyk Instrum. gegrav. Musyk en Ital. Viool Snaaren.

$\mathrm{Nu}$ zijn, wel is waar, de adressen van den magazijn-catalogus uit 1778 en in het adresboek voor 1784 niet gelijkluidend, waar de eerste vermeldt

1) Bchiublekn t. a. p.

2) E. L. Grabrr. Neues Lexikon der Tonkünstler. Zweiter 'Teil. Leipz. 1812. Kul. 713. 
over den eersten bijbel, het andere naast den eersten liesveldschen bijbel, maar ik meen het er toch wel voor te mogen houden, dat J. J. Hummes in de Warmoesstraat te Amsterdam in of omstreeks 1782 geworden is B. Hummel \& Zoon in de Warmoesstraat en zulks omdat de filiaalleider naar Berlijn vertrekkende de zaak overliet aan zijn broeder te 's Gravenhage, die er zijn zoon in plaatste.

Het geciteerde Naamregister voor 1784 kent echter nog een tweeden Hummel:

Hummel, (J. J.) op 't Rokkin over de N. Z. Capel, in Musicale Instrum. en gegrav. Musicalen, \&c.

Het ligt voor de hand dezen Hummes te identificeeren met Johann Julius, die naar Berlijn vertrokken is en in dit adres dus te zien de „vollständige Niederlage seiner Verlagswerke" ${ }^{1}$ ), die door zijn dochter Von Metringh en echtgenoot beheerd werd, m. a.w. dat dit is de plaats van het dusgenoemde groote muziekmagazijn te Amsterdam, dat als depot van de berlijnsche zaak in stand is gebleven nog een aantal jaren er wordt genoemd tot het jaar 1821 - na het overlijden van JoHANN Julius. Johann Julius overleed te Berlijn den 27sten Februari 1798, waar hem den titel van „Königl. Commerzienrath” (Mendel) verleend was.

Burchard overleed te 's Gravenhage 27 September 1797, zijn weduwe, die toen nog in hetzelfde huis in de Spuistraat woonde, den 26sten October $1810^{2}$ ).

De historische beteekenis van Johans Julius Hummel ligt in zijn uitgevers-werkzaamheid. Hoewel hem, volgens GERBER, muziekkennis ontbrak, had hij als handelsman een des te beteren kijk op de mercanteele waarde der toenmalige nieuwe muziekkopijen. Hij wist er heel wat voor zijn fonds te bemachtigen, met name de Symfoniën van Haydn, waarvan hij er sedert 1772 niet minder dan 36 moet hebben uitgegeven.

Zooals vóór hem en nu nog gebruikelijk is, zijn zijn uitgaven immer verschenen zonder vermelding van een jaar van uitgifte, hetgeen de preciese tijdsbepaling ervan uitermate bemoeilijkt. In de plaats daarvan zijn de uitgaven doorgenummerd; het hoogste cijfer, dat ik ken, is nr. 1586, gegeven aan:

\footnotetext{
1) Grrbir a. a. 0 .

2) SCHEURLEkir t. a. p.
} 
Zwaneveld. Variations pour le piano forte, composées et dédié à Mademoiselle la Baronne Agathe de Heeckeren de Kell par M: -, organist et maitre de musique a: Doetinchem. Chez J. J. Hummel à Berlin avec privilege du roi, à Amsterdam au grand magasin de musique et aux adresses ordinaires.

(Verz. J. W. E.)

De verhuizing naar Berlijn maakt zich uiterlijk kenbaar in het uitgeversadres. De oudste mij bekende editie, welke het nieuwe adres vertoont, heeft tot volgnummer 331 en is:

Six trios a violon, alto viola $\&$ violoncello obligé. Ciomposées par Luigı Boccherini, de Lucca. Oeuvre neuvieme. Chez Jean Julien Hummel, à Berlin sous privilége de sa Majesté le roi de Prusse \&c. \&c. à Amsterdam au Gravil Mayasin de Musique et aux adresses ordinaires.

De lagere nummers hebben als adres:

(Verz. (J. W. E.)

a Amsterdam chez J. J. Hсмmfr, marchand et imprimeur au grand magasin de musique, of Marchand et imprimeur de musique.

Van die dus zeer bepaaldelijk amsterdamsche uitgaven bezit ik:

XII Mencets a deux violons et basse, deux clarinettes et deux cors de chasse, exécutés a Berlin a l'occasion de la solemnité du mariage de S. A. S. monseigneur le prince d'Orange, avec S. A. R. Frederique Sophie Wilhelmine, princesse de Prusse \&ic. A Amsterdam chez J. J. Hummel, marchand \& imprimeur de musique. Nr. 82.

Ditgaaf voor klavier 2-handig. - De huwelijksvoltrekking had plaats 4 Oct. 1767. HAyDN, Jos. - Six sonates a deux violons \& basse, dédiés a Monsieur Jean Nepveu, fils ainé de Monsieur Jsac Nepveu à Amsterdam, composés par Giuseppe Haydn à Vienne, opera troisieme. A Amsterdam chez J. J. Hummel, marchand \& imprimeur de musique. Nr. 91.

Titel door A. L. WILdeman 1767.

BoccherinI, Luigi. Six trios a deux violons et violoncello obligé. Composées par -. Oeuvre quatrieme. A Amsterdam chez J. J. Hummel, marchand \& imprimeur de musique. Nr. 202.

HAYDN, J. - Six quatuor a deux violons, taille et basse. Dediés a Monsieur F. G. Stolkert a Paramaribo par Jean Julien Hummel. Composés par Giuseppe Haydn. Oeuvre VII. A Amsterdam chez J. J. Hummel, marchand \& imprimeur de musique. Nr. 208.

Titel door A. L. WiLdxMan. 
Michelet, F. G. De zangwysen der CL. psalmen Davids, en der lofzangen, zo als dezelve in de gereformeerde kerken deser landen gebruikelijk zijn, met voldoende harmonyen voorzien, om op 't orgel of in huis gespeelt te kunnen worden, benevens een voorbericht; opgestelt voor de gene die de basso continuo, niet verstaan, door F. G. Michelet, organist te Alkmaar. By J. J. Hummel, musiekverkooper te Amsterdam. Nr. 250.

Het voorbericht is gedateerd Alkmaer, den 17 Nov. 1771.

Raimond, Ignazio. Six trios a deux violons et violoncello. Composées par -. Oeuvre premiere. A Amsterdam chez J. J. Hummel, marchand \& imprimeur au grand magasin de musique. Nr. 295.

Van gedateerde amsterdamsche uitgaven, zonder uitgeversnummer, zijn in mijn bezit:

Marpurg, F. W. - Aanleiding tot het clavier-speelen, volgens de hedendaagsche luisterryker manier van uitvoering; opgestelt door den beroemden Friedrich Wilhelm Marburg, muziekkundige en componist te Berlyn. Met zeven nooten-tabula's. Uit het hoogduitsche vertaalt en met ophelderende byvoegselen voorzien door $\mathrm{Jacob} \mathrm{W}$ il hel m Lustig, organist van de groote kerk te Groningen. Te Amsterdam, by J. J. Hummel, musiekverkooper in de Nes. 1760.

Neuman, George. De muzicale zangwyzen van het boek der psalmen, nevens de gezangen by de Hervormde Kerk van Nederland in gebruik: volgens de nieuwe dichtmaat en naar de tegenwoordige zingwyze ingericht; om te dienen zo wel voor de stem, als tot gebruik voor muzicale instrumenten. Alles t' samen gesteld en verrykt met een nieuw gecomponeerde bas, om te zingen en te speelen; door -. Te Amsterdam by J. J. Hummel 1776.

Titel door S. Forkx naar W. WRITs.

En van ongedateerde zonder uitgeversnummer, zoo Amsterdamsche als haagsche:

Mahaut, Ant. Nieuwe geöpende musicaale tydkorting bestaende in nieuwe hollandsche zangairen voor een zangstem en basso continuo. Eenige met twee, drie en meer stemmen. Allen zeer bekwaem om op de clavecimbael, viöol en dwarsfluit gespeeld te worden. Door -. 1ste stukie. Met privilegie. Te Amsterdam, by J. J. Hummel. Musijkverkoper in de Nes. 
Pasqual, Nicola.s. De generaal-has gemakkelyker voorgedraagen; of eene beknopte verklaaring van de accoorden. die het clavecymbel hevat; van de heste manier om dezelve samen te voegen; als meede, van de algemeene en byondere regelen, tot het accompagnement van allerhande musiekstukken aanleidende: voorzien met verscheide nooten-exempels. gegraveerd op XIV. plaaten, en bestemd tot het gebruik van aanvangeren. door - . Overgezien en vermeerderd door J a c o b Wilhelm Lustig, organist te Groningen. T'e Amsterdam, by J. J. Hummel, musiekdrukker en verkooper in de Nes.

Tekst fransch en nederlandsch.

De muziekplaten ontbreken; niettemin schijnt het exemplaar compleet.

Manuct, A. Nieuwe manier om binnen korten tyd op de dwarsfluit te leeren speelen, tot gebruik van aanvangers en meer gevorderden opgesteld, door -. Tweede druk. Voorzien met 12 nooten tabula's. Te Amsterdam, by J. J. Hummel, in 't groote musiek magazyn.

Tekst nederlandsch en fransch.

Boutmy. Korte verhandeling over de basso continuo of het kortste en zekerste middel, om dezelve in volmaaktheid te leren. Door de heer -, leermeester op de clavicimbel in 's Hage. A la Haye, chez B. Hummel, marchand \& jmprimeur de musique. On peut l'avoir aussi à Amsterdam chez J. J. Hummel.

Tekst nederlandsch en fransch.

Merchi, G. Sei trio a due violini e basso composti da Giacomo Merchi di Brescia. Opera V. Se tend a La Haye chez Homel et aux adresses ordinaires.

Titel door G. v. MokLINGEN. - Wekt den indruk een duitsche nadruk te zijn.

Van de amsterdamsche en haagsche vertakkingen samen ken ik:

Extrait des airs françois de tous les operas nouveaux qui ont été representés. Appropriés pour le chant ou la flute avec la basse continue. Partie I [-V]. A Amsterdam chez J. J. Hummel, à La Haye chez B. Hummel, marchands \& imprimeurs de musique. (Verz. J. W. E.)

Titel van partie I door A. L. WILdemaN, van partie V door C. J. Dx Hurser.

Sterkel, J. F. Trois sonates pour le clavecin ou piano forte avec l'accompagnement d'un violon obligé composées par - . Oeuvre XVIII. Libro I. A La Haye et à Amsterdam chez B. Hummel et fils.

(Verz. J. W. E.) 
Journal de La Haye ou choix d'airs firançais dédié aux dames. Libro I. A la Haye et à Amsterdam chez B: Hummel et fils. (Verz. J. W. E.) Wittenberg, F. J. Six trios à deux violons et violoncello composés par -. Oeuvre II. A La Haye et à Amsterdam chez B: Hummel et fils.

(Verz. J. W. E.)

Al deze uitgaven, zonder uitzondering, zijn, wat de noten betreft, uitgevoerd in plaatdruk. Niet alleen daarom, maar ook om het jaartal, kan ik de volgende uitgaaf niet te best verklaren.

Lieder mit Melodien. Herausgegeben von Johann Matrheus König, Königl.

Preuszl. Kammer-Canzellist zu Ellrich. Berlin, 1778. Zu finden in der königl. privilegirten Musikalien Stecherey und Handlung, bey J. J. Hummel.

(Verz. J. W. E.)

Deze uitgaaf is uitgevoerd in boekdruk, volgens het muziek-zetsysteem van BREITKopf, wat een eenigszins vreemd aspect geeft te midden van deze uitgebreide serie van tindrukken, des te zonderlinger, omdat Hummer hier uitdrukkelijk de aandacht vestigt niet op zijn muziek-boek-, maar wel op zijn muziek-plaatdruk. Bovendien past het jaartal 1778 niet in het verband hierboven ontwikkeld, omdat in den magazijn-catalogus hierachter gezegd wordt, dat hij in dat jaar in Amsterdam en niet in Berlijn gevestigd was, en ik mede op grond daarvan zijn verplaatsing naar Duitschland in of omstreeks 1782 meende te moeten stellen.

Ook biedt bibliografische bezwaren:

MARPURG, F. W. Fughe e capriccj pe l'clavicembalo ò per l'organo composti e dedicati al celebre signore G. P. E. Bach, dal suo servo, ed amico -. Opera prima. Chez Jean Julien Hummel; à Berlin avec privilége du roi, à Amsterdam au grand magazin de musique et aux adresses ordinaires. Nr. 153.

(Verz. J. W. E.) omdat het catalogus-nummer van dezen berlijnschen druk ligt te midden der amsterdamsche volgnummers, en de titel genoemd wordt op den hierachter volgenden magazijn-catalogus van de amsterdamsche en haagsche branches. De uitgaaf komt hierin dus overeen met de hiervoor geciteerde trios van BoccherinI, reden waarom het ten slotte toch wel mogelijk kan zijn, dat JoHANN JuLus in 1774 naar Berlijn verhuisd is.

Die magazijn-catalogus, een tweezijdig bedrukt planoblad in de Bibliotheek van de Vereeniging ter Bevordering van de Belangen des Boek. 
handels te Amsterdam, heb ik gemeend zonder eenige commentaar hier te mogen afdrukken zonder daarin eenige correctie, welke ook, aan te brengen. Vandaar dat slordigheden in orthografie en in interpunctie behouden zijn. Ik deed zulks, omdat deze druk daarmede in schrille tegenstelling is met de doorgaans nauwkeurige correctie van HummeL's muziekuitgaven en die incorrectheid een wellicht niet onbelangrijken kijk geeft op het handelshegrip van dezen duitschen muziekdebitant. Het was waarlijk niet noodig geweest, dat deze fransche druk zoo slecht verzorgd is; zoowel in Amsterdam als in Den Haag waren destijds behoorlijk ingerichte fransche boekdrukkerijen; in Amsterdam bezorgden de uitgevers Rey, Van Harrevelt en Gúrin, in Den Haag Neaulme en Gosse typografisch voldoende verzorgde kopijen ter perse.

De catologus is, naar het mij toeschijnt, een niet geheel onbelangrijke bijdrage voor de muziekbibliografie in het algemeen, voor de wetenschap, wat een goed voorziene assortimentshandel in 1778 in voorraad meende te moeten hebben in het bijzonder. De uitgaven van Burchard HummeL alleen zijn blijkens een aanteekening in deze titellijst gemerkt met een sterretje.

J. W. ENSCHEDÉ. 


\section{CATALOGUE,}

DES LIVRES DE MUSIQUE TANT VOCALE QU'INSTRUMENTALE. QUI SE VENDENT CHEZ JEAN JULIEN HUMMEL, MARCHAND

AU GRAND MAGAZIN DE MUSIQUE DANS LA WARMOES-

STRAAT, VIS A VIS LA PREMIÈRE BIBLE À AMSTER-

DAM, ET CHEZ BURCHARD HUMMEL, AU GRAND

MAGAZIN DE MUSIQUE DANS LA SPUY.

STRAAT, À LA HAYE, 1778.

Catalogue thématique, ou commencement des Oeuvres suivantes, pour la Commodité des Amateurs, afin de voir tout d'un coup, si les Pieces qu'on leur presente en Manuscrit, n'ont pas deja éte imprime, proprement gravé, du premier jusqu'au septième Supplement, inclusif pour le prix de... . . . . . . . . . . . . . . 8 5.-.

\section{Simphonies ou Overtures à 8 Parties.}

fl. sols

Abel, 6 Simph. a 8 parties Hautbois \& Cors ad lib. op. 1. . . 6 -- 6 Simph. a 8 part. op. 7. . . . . . . . . . . . . 6

- 6 Simph. a 8 part. op. 10 . . . . . . . . . . . . 6 -

Aleuni Fam. Maestri, 6 Simph. a 8 part. . . . . . . . . . 6 -

Bach, 6 Simph. a 8 part. op. 3 . . . . . . . . . . . . 6 --

- 6 Simph. a 8 part. op. 6. . . . . . . . . . . . . 6 -

*__ 3 Simph. a 8 part. op. 9. . . . . . . . . . . . . 3-10

*Daraux, 2 Simph. Conc. op. 3. . . . . . . . . . . . . 4-

*_- 2 Simph. Conc. op 4 . . . . . . . . . . . . . . 4:

*-2 2 Simph. Conc. op 5 . . . . . . . . . . . . . . 5-

*Eichner 3 Simp. chois. a 8 part. op. 7. . . . . . . . . . 4:10

*__, 3 Simph. a 8 part. op. 8. . . . . . . . . . . . . $4-10$

*Filtz, 6 Simph. chois. a 8 part. . . . . . . . . . . . . 6 -

Glaser, 6 Simph. a 8 part. op. 1 . . . . . . . . . . . . 6- 
Gloesch, Marche la Garde passe, varié pour le Clavecin, la Flute,

Violons, Viola et Violoncello.

Graar, 6 Simph. a 8 part. op. 7

- 6 Simph. a 8 part. op. 9 .

- 6 Simph. a 8 part. op. 11.

-_ 6 Simph. a 8 part. op. 14.

- 6 Simp. a diverse Instr. op. 16.

Greiner, 6 Simph. a 8 part. op. 2. $6-$

Haydn, 3 Simph. a 8 part. op. 10.

Hupfeld, 6 Simph. a 8 part. op. 3.

Kammel, 6 Simph. op. 11. a 8 part.

Kloffier periodique Simph. No. I, II. et III. chaqu'un .

Kreilsser, 6 Simph. a 8 part. op. 2

-..- 6 Simph. a 8 part. op. 5 . $6-$

6 Simph. a 8 part. op. 7 . $6-$

6 Simph. a 8 Part. op. 9 . $6-$

- 3 Simph. a 8 part. op. 13. au mois de Juillet 1778 $6-$

-- 1 Simph. Trom. \& Timb. $4-$

Kospoth, (Baronde) 3 Simph. op. 1. 1778.

Meder, 6 Simph. a 8 part. op. 1 $3-10$

Ouverture de la Rosiere a 8 part. 6 -

* Ouverture Détaché du Déserteur a 8 part. 1-10

*Ouverture de Henry IV. ou la Bataille d'Ivry, a 8 part. . . . 2 -

*Entr'Acte du même opera a 8 part. . . . . . . . . . . 1-5

*Ouverture de la belle Arséne, a 8 part. . . . . . . . . . 1-10

Pichl 6. Simph. a grand Orchestre op. 1. au mois de May. $17786-$ Récreat. d'Apollon ou 3 Simph. a 8 part tiré des Opera Français, Part. I . . . . . . . . . . . . . . . . . . 4-

Richter, 6 Simph. a 8 part. op. 2. . . . . . . . . . . . $6-$ - 6 gr. Simph. op. 4 . . . . . . . . . . . . . . .6--

Ricel, 6 Simph. a 8 part. op. 2 . . . . . . . . . . . . 6 Sehmitt, (J.) 6 pièces cons. en 2 quart, 2 quint. \& 2 Simph. op. 16 - 6 gr. Simph: a 8 part. op. 6. . . . . . . . . . . . 6

Stamitz 6 Simph. choisies . . . . . . . . . . . . 6 - 
Stamitz. (Charles) 2 grand simph. concerlantes a 10 piat. op. 24

2 gland simph. concertanles a 10 palt. op. 3 . . . 4

..... 2 gl. Simp. conc. a 10 p. op. 4 . . . . . . . . . . 4

... 6 simpl. a 8 part. op. 9 . . . . . . . . . . . . . 6

.... 6 s.imph. a grand Orchestre. op. 13. . . . . . . . . 6

*Schwindl. 6 Simph. a 8 part. op. 1 . . . . . . . . . . . 6

*... 6 simph. a 8 part. op. 2. . . . . . . . . . . . . 6

*... .. , 3 Simph. a $\$$ part. op. 9. . . . . . . . . . . . . 4

Toeschi, 6 gr. Simph. a 8 part op 4 . . . . . . . . . . . 6

Zingoni, 8 Simph. a 8 part. op. 1. . . . . . . . . . . . 7

Divers. Auteurs, 6 symph. a 8 part. comme Toeschi, Schwind,

Klöffler, Ditters, Kreüsser \& Schweizer, opera 1 . . . . . $\mathbf{6}$ -

Divers. Auteurs, 6 simph. a 8 part. comme Hartman, Ditters,

Toeschi, Ditters, Schmitt \& Ditters, opera 2 . . . . . . . $\mathbf{6} \ldots$

Divers. Auteurs, 6 simph. a 8 part. comme Haydn, Toeschi,

Klöffler, Toeschi, Ditters \& Schmitt, opera 3. . . . . . . 6

Divers. Auteurs, 6 Simph. comme Greiner, Masch, Schmitt,

Vanhal, Klöffler \& Haydn, a 8 part. op. 4. . . . . . . . 6

Divers. Auteurs, 6 Simph. a 8 part. comme Klöffler, Haydn,

Vanhal, Bach, Vanhal, Toeschi, op. 5. . . . . . . . . . 6 -...

On vend cette Simph. Périod. séparem. a. . . . . . . . 1-10

La continuation sera.

\section{Quintets et Quartets à Flute, Violon, Alto \& Basse.}

Abel, 6 quatuor op. 8 . . . . . . . . . . . . . . . . 5-

-. 6 quat. Flute, \& Violon. op. 12. . . . . . . . . . 5-

Bach, 6 quat. Flute \& Violon, op. 8. . . . . . . . . . . 5-

- 6 quintets, 2 Flutes \& Violon, op. 11 . . . . . . . . 5-

_-_, Abel \& Giardiui, 6 Flute ou Violon quatuor . . . . . 5-

Boccherini, 6 quatuor op. 1 . . . . . . . . . . . . . . 5-

- 6 quatuor op. 2 . . . . . . . . . . . . . . . . 5 -

- 6 quatuor op. 7 . . . . . . . . . . . . . . . . 5 -

- 6 quatuor ou Divert. op. 8 . . . . . . . . . . . . 4 -

Cambini, 6 quat. Fl. \& Viol. op. 1 . . . . . . . . . . . 5- 
*Cambini, 6 quatuor op. 3 .

*Canabich, 6 quat. Flute, \& Viol. op. 1 . . . . . . . . . . . 4-

Cirri Sextetto . . . . . . . . . . . . . . . . . . . 1-10

*Daraux, 6 quatuor op. 1 . . . . . . . . . . . . . . . 4 -

Eichner 6 quat. Flute $\mathbb{E}^{\mathrm{N}}$ Violon, op. 4 . . . . . . . . . . 5

Fatken, 6 quat. Flute \& Viol. op. 1 . . . . . . . . . . . 4-

Gassman, 6 quatuor, op. 1 . . . . . . . . . . . . . . 5 --

Gossec, 6 quatuor, op. 1 . . . . . . . . . . . . . . . 5 -

Graaf, 6 quintets, Flute, Viol. Alto, Violone. \& Basse, op. 8 . . 5-6 quat. Flute \& Violon, op. 12. . . . . . . . . . . 5

_-6 quat. Violone. obl. op. 35. . . . . . . . . . . . 5-

Haydn, 6 quatuor op. 1 . . . . . . . . . . . . . . . 5-

-6 quatuor op. 2 . . . . . . . . . . . . . . . . 5-

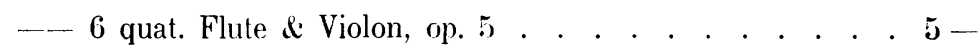

-6 quatuor op. 7 . . . . . . . . . . . . . . . . 5

6 grand quat. op. 9. . . . . . . . . . . . 6-

Heinsius, 6 aisée Simph. a 4 part. op. 2 . . . . . . . . . 4

Hoffstetter, 6 quatuor op. 1. . . . . . . . . . . . . . 5

Kammel, 6 quatuor op. 4. . . . . . . . . . . . . . . 5

-6 6 Quatuor op. 8. . . . . . . . . . . . . . 5

kreusser, 6 quat. Flute \& Viol. op. 8 . . . . . . . . . . 4 --

6 quint. Flute \& Viol. op. 10 . . . . . . . . . . . $6-$

6 quart. op. 12. au mois de juin 1778 . . . . . . . . 5-

*Lorenziti, 6 quatuor op. 1 . . . . . . . . . . . . . . 5-

Michaelis, 6 quatuor op. 1 . . . . . . . . . . . . . 5-

Pagnanl, 3 quatuor . . . . . . . . . . . . . . . . . $3-$

- 3 quintets. . . . . . . . . . . . . . . . . 4-10

- 3 quintets, op. 7 . . . . . . . . . . . . . . . . 4-10

Raimondi quatuor, Flute \& Violon . . . . . . . . . . . 1 -

Rigel, 6 quat. Ful. et Viol. op. 1 . . . . . . . . . . . 5 -

Schmitt (J.) 6 quat. Flute \& Viol. op. 3 . . . . . . . . . 5 -

--, 6 quatuor op. 5 . . . . . . . . . . . . . . . . 5-

Stamitz (Charles) 6 quat. a une Clarinette (Flute ou Hautbois)

Violon, Alto, (ou Cor de Chasse) \& Violone. op. 8. . . . . 5- 
*Stamitz (Charles) 6 quart. a 2 Tailles Violon \& Violonc. op. 105 : (C.) 6 quat. 2 a grand Orchestre 2 Concert. \& 2 a Flut. ou Houtb. op. 11

*Schwindl 6 quatuor op. 7 .

Solnitz 6 Simph. a 4 part. op. 3

Toëschi 6 quat. Flute \& Violon op. 1

- 3 quart. \& 3 quint. op. 3 .

-_ 6 quart. op. 5.

Vanhal 6 grand quat. op. 3 .

*Wondling quat. de Lucile avec l'Allern.

$1-$

\section{Concerts pour le Violon.}

Bach, Concert ou Simpl. concertante a plusieurs Instrum. . . 2 -

Borghi 3 Violon Cioncerts op. 2 . . . . . . . . . . . . 4-10

3 Viol. Concert op. 3 . . . . . . . . . . . . . . 4-10

*Colizzi Viol. ou Mandolino Cioncert . . . . . . . . . . . 1-10

*Daranx Viol. Conc. op. 2 N0. 1 . . . . . . . . . . . . 2 -

*__ ditto - $\mathrm{N}^{0} .2$. . . . . . . . . . . . . . . . . 2 -

Ernst Viol. Conc. op. 1. . . . . . . . . . . . . . . . 1-16

Fischer, Viol. Flut. ou Oboe Cionc. . . . . . . . . . . . 2

Glardini, 3 Viol. Cionc. op. 4 . . . . . . . . . . . . . 4-10

—- 3 Violon Conc. op. 5 . . . . . . . . . . . . . . 5-

Morigi, 6 Violon Concerts avec les agrements, op. 3. . . . . 7 -

Nardini, 6 Violon Conc. op. 1 . . . . . . . . . . . . . . 7 -

Raimondi, Viol. Conc. a 8 part. op. 2. lib. 1. . . . . . . . 1-16

—_ ditto lib. II . . . . . . . . . . . . . . . . . . 2

Sirmen (Mad.) 3 Viol. Conc. op. 2 . . . . . . . . . . . 4-10

— 3 Viol. Conc. op. 3 . . . . . . . . . . . . . . . 4-10

Puel, Violon Concert . . . . . . . . . . . . . . . . 1-10

\section{Concerts pour la Flute Travers.}

Besozzl, Flute Trav. Conc. . . . . . . . . . . . . . . 1-10

Eichner, Oboe ou Flute Concert . . . . . . . . . . . . 2 -

Gloesch, 3 Flute Concerts, chaqu'un . . . . . . . . . . . 1-10

Les sujets s'ont tiré des quelques Airs Franç. 
Graaf (F. H.) 3 Fl. Trav. Conc. op. 4 . . . . . . . . . . . 4-10

Hnpeld Flute Travers Concert. . . . . . . . . . . . . 1-16;

Leeder Flute Travers Cioncert . . . . . . . . . . . . . 1-16

Kloffler, 6 Fl. Trav. Cione. 3 a un \& 3 is 2 Fl. op. 1 . . . . 8 -

- 3 Fl. Trav. Conc. op. 9 . . . . . . . . . . . . . 4

- 3 Conc. à 2 flutes trav. op. 3 . . . . . . . . . . . 4 -

Reinatds, 6 Fl. Trav. Conc. op. 3. . . . . . . . . . . . 7 -

Tromlitz, 3 Flute Conc. op. 1 . . . . . . . . . . . . . 4-

\section{Concerts, Simphonies \& Quatuor pour le Clavecin.}

Abel, 6 Concerts op. 11 . . . . . . . . . . . . . . 6-

Bach, (J. C.) 6 Conc. op. 1 . . . . . . . . . . . . . . 7 -

-- 6 Con. op. 7 . . . . . . . . . . . . . . . . . 7 -

*Bach, 3 Conc. op. 12 . . . . . . . . . . . . . . . . . . 4-10

Bach, 3 Conc. op. 13 . . . . . . . . . . . . . . . . . 4-10

Bach, (J. M.) 6 Aisée Cionc. op. 1 . . . . . . . . . . . . 7 -

*Collzrs, concert. . . . . . . . . . . . . . . . . . . 1-10

Ditters, Conc. . . . . . . . . . . . . . . . . . . . 2-10

Eichner (J. T.) 2 gr. Concert. op. 5 . . . . . . . . . . . 4 -

Glordanl 3 Clavier Concerts op. 12 . . . . . . . . . . . 4-

3 Clavier concerts op. 13, 1778. . . . . . . . . . . 4-

Herschel, 6 quat. 2 Viol. \& Violonc. op. 1. . . . . . . . . $6-$

Kelner, Clav. Cionc. . . . . . . . . . . . . . . . . . 2-

Kreusser, Simph. av. un Viol. op. 6 . . . . . . . . . . . 1 -

Relchardts, 6 conc. pour le beau Sexe op. 1. . . . . . . . 7 -

Sehobert, 3 quat. av. 2 Viol. op. 2 . . . . . . . . . . . 4-

- 3 Simph. avec l'accomp. d'un Viol. \& Cors ad lib. op. 5 . 3 -

- 3 ditto op. 6. . . . . . . . . . . . . . . . . . $3-$

-- grand Conc. lib. I. . . . . . . . . . . . . . . . $3-$

- ditto lib. II . . . . . . . . . . . . . . . . . . 3 -

- ditto lib. III . . . . . . . . . . . . . . . . . . $3-$

- ditto lib. IV . . . . . . . . . . . . . . . . . . $3-$

- ditto lib. V. . . . . . . . . . . . . . . . . . $3-$

- ditto lib. VI. . . . . . . . . . . . . . . . . 3- 
Schröter, 3 Concerts op. $4.4-$

-. 3 Concerts op. 5. . . . . . . . . . . . . . . . 4 -

Schultz 6 quatuor op. 1 . . . . . . . . . . . . . . . . . . 5 -

*Wagenseil, Concert choisie . . . . . . . . . . . . . . 2-5

*Stamitz, Concert . . . . . . . . . . . . . . . . . . 2-5

*Rusch, ditto lib. 1. . . . . . . . . . . . . . . . . . 1-10

*__ ditto lib. 2 . . . . . . . . . . . . . . . . . . 1-10

*Manfredini, ditto . . . . . . . . . . . . . . . . . . 2-10

\section{Trios pour le Clavecin.}

Abel, 6 trio con Violon ou Flute op. 5. . . . . . . . . . 4-10

*... 6 trio avec Viol. op. 13. . . . . . . . . . . . . . 4 -

Bach, 6 trio avec Violon op. 2. . . . . . . . . . . . . 5-

- 6 trio avec Violon, op. 10. . . . . . . . . . . . . . 4 -

__. (C. P. E.) 6 Trios op. 2. 1778 . . . . . . . . . . . . . 5 -

Boccherini, 6 gr. trio avec Viol. op. 5 . . . . . . . . . . 5 -

*Boutmy, 6 Divert. Viol. ad lib. . . . . . . . . . . . . . 3-10

Chalon, 6 trio, Viol. ad lib. op. 2. . . . . . . . . . . . 5-

*Colizzi, 3 trio avec Viol. op. 3 . . . . . . . . . . . . 2-10

*__ 3 trio avec Viola op. 4. . . . . . . . . . . . . . 2-10

Dietz, 6 trio avec Viol. \& Violonc. op. 1 . . . . . . . . . 5-

Eichner, 6 trio avec Viol. \& Violonc. op. 1 . . . . . . . . 6 -

- 3 trio avec Viol. \& Violonc. op. 2. . . . . . . . . . 3 -

- 3 trio avec Viol. \& Violonc. op. 2. . . . . . . . . . 3 -

Filtz, 6 trio avec Viol. op. 2. . . . . . . . . . . . . . 5-

Graaf, 6 trio avec Viol. op. 4 . . . . . . . . . . . . . 5 -

—— 3 trios avec Violon op. 13 . . . . . . . . . . . . 3 -

Haueisen, 3 trio avec Violon op. 1 . . . . . . . . . . . 2-10

Haydn, 6 trio avec Viol. op. 4 . . . . . . . . . . . . 5-

Just, 6 Divert. avec Viol. op. 1. . . . . . . . . . . . . 4-

*_- 6 trio avec Viol. op. 2 . . . . . . . . . . . . . . 5-

- 6 Sonatin. Viol. ad lib. op. 4 . . . . . . . . . . . 2

*_ 6 Divert. avec Viol. op. 6. . . . . . . . . . . . . 4-

Kammel 6 trio Viol. \& Violonc. op. 10 . . . . . . . . . 5 - 
H. sols

Leeder 6 trio arer Viol. op. 1 . . . . . . . . . . . . . . 4

Michaelis, 6 trio aver Violon. op. 2. . . . . . . . . . . 5

*Mozart, 6 trio aver Viol. op. 4. . . . . . . . . . . . . 3

Neuman 3 Aisée sonatines avec 2 Viol. di Violonc. op. 1, 1778. 3

-- 3 Sonates avec 2 Viol. \& Violonc. op. 2, 1778 . . . . . 4

-..-. Overture de la Belle Arséne, arer un Violon. . . . . . - -15

- - Ouverture du Huron, $d^{0}$. . . . . . . . . . . . . . $\quad-15$

-- Ourerture du tableau parlant. $d^{0}$. . . . . . . . . . -15

....- Ouverture de Henry IV, $\mathrm{d}^{0}$. . . . . . . . . . . . 1 -

- Overture de la Rosiere, 1778. . . . . . . . . . . . $\quad-15$

Paganelli, 6 Divert. avec Viol. . . . . . . . . . . . . . $3-$

Pugaani 6 trio av. Viol. \& Violonc. op. 6. . . . . . . . . 5 -

Reichardts 6 trios aver: Violon, op. 2 . . . . . . . . . . 4 -

Richter, 4 trio, op. 1 . . . . . . . . . . . . . . . . 3-10

*Rusch, 6 trio op. 3 . . . . . . . . . . . . . . . . . 4-

*Rappe 4 trio avec Violon op. 1. . . . . . . . . . . . . 4-

Sarti, 3 trio avec Violon . . . . . . . . . . . . . . . 2-10

*Schmid, (J. B.) 6 trio, op. 3. . . . . . . . . . . . . . . 4-

Schröter 6 trio av. Viol. \& Violonc. op. 2. . . . . . . . . 4-

*_- 6 trio avec Flute op. 6. . . . . . . . . . . . . . 3-10

Sehobert, 3 trio avec Viol. op. 1 . . . . . . . . . . . . 4-

4 trio âvec Viol. \& Violonc. op. 3. . . . . . . . . . 5-

- 6 trio avec Viol. op. 4 . . . . . . . . . . . . . 5-

- 4 trio avec Violon op. 7 . . . . . . . . . . . . . 4-

- 4 trio av. Viol. op. 8 . . . . . . . . . . . . . . 4-

Schwindl, 4 trio avec Viol. \& Violoncel. op. 8 . . . . . . . $3-$

Uber, (Mr.) 3 trio avec Viol. op. 1 . . . . . . . . . . . 2-10

- 6 Sonatirus, and Viol. op. 2, 1778. . . . . . . . . . 1-16

\section{Trios à 2 Violons \& Basse.}

Abel, 6 trio op. 3. . . . . . . . . . . . . . . . . . 3-

Bach, 6 Ajsée trio op. 4 . . . . . . . . . . . . . . . 3-

Abel en Kammel 6 Trios, 1778. . . . . . . . . . . 3-10

Benda (Fr.) 6 Trio op. 1 au mois d'Avril, 1778 . . . . . . 3-10 
Boccherini 6 trio op. 4. . . . . . . . . . . . . . . . 3-10 -- 6 trio op. 6 . . . . . . . . . . . . . . . . . . . . . $3-10$ 6 trio, Violon, Viola \& Violoncello obl. op. 9. . . . . . 4_- 6 trio Violonc. obl. op. 10. . . . . . . . . . . . . 3-10

Grann 6 trio op. 1 . . . . . . . . . . . . . . . . . . 3-10

Camploni, 6 Trio op. 6 . . . . . . . . . . . . . . . . 3-10

— op. 7. . . . . . . . . . . . . . . . $3-10$

Cramer, 6 trio, op. 1 . . . . . . . . . . . . . . . . $3-10$

- 6 trio op. 2 . . . . . . . . . . . . . . . . . . 3 -

Filtz, 6 grand trio op. 3 . . . . . . . . . . . . . . . 4

Fransechini, 6 trio op. 1 . . . . . . . . . . . . . . . 3-10

Galliotri, 6 trio op. 2 . . . . . . . . . . . . . . . . 3-10 6 Trio, op. 3 . . . . . . . . . . . . . . . . . 3-10

Giardini 6 trio Violon, Viola \& Violoncello op. 2 . . . . . . 3-10

Graaf, 6 trio op. 10 . . . . . . . . . . . . . . . . . 3-10

Guerini, 6 trio op. 6. . . . . . . . . . . . . . . . . $3-10$

- - op. 7. . . . . . . . . . . . . . . . . 3-10

Haydn, 6 trio, op. 3. . . . . . . . . . . . . . . . . 3-10

— 6 trio op. 8 . . . . . . . . . . . . . . . . . . 3-10

— 6 aisé trio op. 12. . . . . . . . . . . . . . . . 2-1

Hoftman, 6 trio, Violon Viola \& Violonc. op. 2. . . . . . . 3 -

Hupfeld, 6 aisée trio op. 2 . . . . . . . . . . . . . . 3-10

Krellsser, 6 aisée trio, op. 1. . . . . . . . . . . . . . 3 -

— 6 trio op. 11 . . . . . . . . . . . . . . . . . $3-10$

*Kammel, 6 trio op. 1 . . . . . . . . . . . . . . . . $3-$

— 6 trio op. 3. . . . . . . . . . . . . . . . . . 3-10

- 6 aisée trio op. 6. . . . . . . . . . . . . . . . 3 -

*_- 6 trio op. 7 . . . . . . . . . . . . . . . . . . 3-10

Nofer, 6 trio 3. a Viol \& viola op. 3 . . . . . . . . . . 3-10

Pugnani, 6 trio op. 3 . . . . . . . . . . . . . . . 3-10

Raimondi, 6 trio op. 1. . . . . . . . . . . . . . . . 3 -

— 6 trio a Violon, Viola \& Violonc. op. 5. . . . . . . . 3-10

*Bicci, 6 trio op. 3 . . . . . . . . . . . . . . . . . 3-10

Blchter, 6 grand trio op. 3 . . . . . . . . . . . . 4- 
Rickert (Eater) 6 divelt: op. 1. all mois atoul. 1778 . . . . 3-10

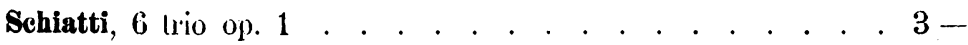

Sehmitt (J.) 6 trio op. 2 . . . . . . . . . . . . . . . . . . . $3-10$

_.. 6 trio op. 4. . . . . . . . . . . . . . . . . . 3-10

*Schwindl, 6 trio op. 5. . . . . . . . . . . . . . . . . . 4-

Sirmen 6 trio op. 1 . . . . . . . . . . . . . . . . . . . . $3-10$

-... (Madame) 6 trio op. 1. . . . . . . . . . . . . . . 3-10

Schuman, 6 trio, Viol. Flute \& Basso op. 1 . . . . . . . . 3-10

*appa, 6 trio . . . . . . . . . . . . . . . ... . . 3 -

Vanhal 6 trio a Serenade, Violon. Viola \& Basso, deux Ciors de chasse ad lib. op. 1 . . . . . . . . . . . . . . . . 46 lrio op. 2. . . . . . . . . . . . . . . . . . 3-10

*Zemire d Asor Trio delaché. . . . . . . . . . . . . . 12

Zebro \& Spang. 12 trio. . . . . . . . . . . . . . 5-

\section{Trios a deux Flut. Travers. \& Basse.}

Campioni, 8 trio op. 4. . . . . . . . . . . . . . . 4-10 Filtz, 6 trio, op. 4 . . . . . . . . . . . . . . . . . 3 -

Giordani 6 trios, Flute, Viola \& Violonc. op. 1. . . . . . . 4 :

Gloesch, 6 trio op. 1 . . . . . . . . . . . . . . . . 3-10

Graaf 6 trio op. 3 . . . . . . . . . . . . . . . . . 3 -

Groneman, 6 trio op. 2. . . . . . . . . . . . . . . . $3-$

Grenser, 6 Trios op. 1. . . . . . . . . . . . . . . . 3 :

Haydn 6 trio op. 11. . . . . . . . . . . . . . . . $3-$

Klofiler, 6 trio op. 5. . . . . . . . . . . . . . . . . 3-10

Muller, 6 trio op. 1. . . . . . . . . . . . . . . . . 3 -

Schwind, 6 trio op. 3 . . . . . . . . . . . . . . . . . 3-10

Stamitz, (C.) 6 trio op. 14 . . . . . . . . . . . . . . . . . 3-10

Wendling, 6 trio, op. 1. . . . . . . . . . . . . . . . 3-10

— 6 trio op. 2 . . . . . . . . . . . . . . . . . . $3-$

— 6 trio op. 3 : . . . . . . . . . . . . . . . . . 3-10

- 6 trio op. 5. . . . . . . . . . . . . . . . $3-$ 
Boccherini. 6 Duo op. :3 $: 3$

Borghi, 6 buos, op. 4 . :3

Cambini 6 Duos op. 2 . 3 :

Canabich. \& Duo. Flute d Viol. op. 2 $2 \cdot 10$

*Colizzi, Airs Franc. en Duo. $1-10$

Francisconi 6 Duo op. 1 3

Francechini, 6 Duo op. 2 . 3

Fritz, 6 Duo 2-10

Frischmuth, 12 Airs culv. V. 2 .

Giardini, 6 Duo op. 3 . 3

Guerini. 6 Dua op. 4 . 3 op. 5 $3 \ldots$

- 6 Duo pour les commenc. op. 10. . . . . . . . . . . . 2

Haydn, 6 Duo op. 6. . . . . . . . . . . . . . . . . . . 3

Huber, 6 Duo pour un Viol. \& Viola op. 1 . . . . . . . . 2-10

*Kammell, 6 Duo op. 2. . . . . . . . . . . . . . . . 3 -

*_- 6 Duo op. 5. . . . . . . . . . . . . . . . . . : 3-

Kerntl, 6 Duo op. 1. . . . . . . . . . . . . . . . . 3 - .

Kretisser, 6 Divert. op. 3. . . . . . . . . . . . . . . 1-10

- 6 Duo op. 4. . . . . . . . . . . . . . . . . . 2

${ }^{*}$ Noferi, 6 Duo . . . . . . . . . . . . . . . . . . . . . . . . $3-$

Pugnani, 6 Duo op. 4 . . . . . . . . . . . . . . . . . . . . $3-$

Raimondi, 6 Duo, op. 4 . . . . . . . . . . . . . . . . . 3 -

Sehmitt, (Joseph) 6 Duo op. 7 . . . . . . . . . . . . . . . . . 3 -

Stamitz, (C.) 6 Duo a Viol. \& Viola op. 1. . . . . . . . . . $3-$

Stamitz 6 Duo a Viol. \& Viola op. 12 . . . . . . . . . . 1-16

*Schlatti 6 Duo. . . . . . . . . . . . . . . . . . . $2-10$

*Sirmen (Mad.) 6 duo op. 4 . . . . . . . . . . . . . . . $3-$

Spadina, 6 Duio op. 6 . . . . . . . . . . . . . . . . . . . $3-$

*Schwindl, 12 Divert. op. 4 . . . . . . . . . . . . . . . . $3-$

\section{Duos pour la Flute Traversiere.}

Dottel Figlio, 6 Duo . . . . . . . . . . . . . . . . 3-

*Dirertiss. Militair, pour 2 Fl. Trav. . . . . . . . . . . -16 
Fischer, 6 (luo. . . . . . . . . . . . . . . . . . $1-16$

Greiner 6 Duo op. 1 . . . . . . . . . . . . . . . . . . . . . $2-10$

Hoffman, 6 Duo o!n. 1 . . . . . . . . . . . . . . . . . . 3

Kerntl. 6 Duo op. 2 . . . . . . . . . . . . . . . . . . $1-16$

Klöffler. 6 Duo op. 4. . . . . . . . . . . . . . . . 3

Pla 6 Duo op. 1 . . . . . . . . . . . . . . . . . . . . . . . . 2

Reinards. 10 Гun pou les rommencens op. 1 lih. 1. . . . . 1-16

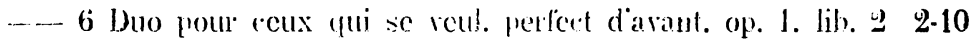

-.., 6 Sonalines op. 4 . . . . . . . . . . . . . . . 2

Stechwey 6 J)uo. . . . . . . . . . . . . . . . . . 3 -

Simono, dirs Franr. cu Dun lih. 1 . . . . . . . . . . . 2-

Fclowindl Avis Franc. en Duo lit. 1. . . . . . . . . . . 1-16

Valentini, \& Juo pour les aprentils op. 1. . . . . . . . . 2-10

Wendling 6 Duo op. 4. . . . . . . . . . . . . . . . . 2 .1

Wolf, ij duo op. 1. 1778 . . . . . . . . . . . . . . . $3-$

\section{Solos \& Duos pour le Violon \& Violoncello.}

Baretté 6 Duo a deux Violonc. pour les commencens. . . . 1-10

Campioni 6 Dun op. 8. . . . . . . . . . . . . . . . . . . 3 -

Cirri 3 Duo op. 1 . . . . . . . . . . . . . . . . . . . . 1 .10

Graziani. 6 Solos, op. 3 . . . . . . . . . . . . . . . 4 --

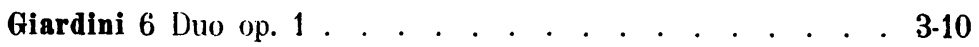

Yojeri, 6 duo op. 2. . . . . . . . . . . . . . . . . . . . 3 -

Schröter, 6 Duo op. 3. . . . . . . . . . . . . . . . 3-

*Schetky, 6 Duo op. 1 . . . . . . . . . . . . . . . . $3-$

*Schwindl, 6 Duo op. 6. . . . . . . . . . . . . . . . 310

\section{Solos à Violon \& Basse.}

Abel, 6 Solos op. 9 . . . . . . . . . . . . . . . . . . . . . . 3

Borghi, 6 Solos op. 1. . . . . . . . . . . . . . . . 3-10

Ferrari, 6 Solos op. 2. . . . . . . . . . . . . . . . 2-10

Hupfeld, 6 aisée Solos op. 1 . . . . . . . . . . . . . 3 -

*Kammel, 6 Solos, op. 9 . . . . . . . . . . . . . . . . . . 4-

Lolli, 6 grand Solos op. 1 . . . . . . . . . . . . . . 4- 
Lolli, 6 Solins op. ㄴ. . . . . . . . . . . . . . . . . . . .

-... isolos d un Divert. op. 3 . . . . . . . . . . . . . 4 -

Nardini. 6 Solos. op. 2 . . . . . . . . . . . . . . . 4

*Pesch, 6 Solns op. 1 . . . . . . . . . . . . . . . . . . 3 -

Pugnani, 6 Solos, op. 5 . . . . . . . . . . . . . . . 4

-. 6 Solos, op. 8 . . . . . . . . . . . . . . . . . 4-

Haimondi, 6 Solos op. 3 . . . . . . . . . . . . . . . . . 4

6 Solss op. 6.1778 . . . . . . . . . . . . . . . 4-

Tartini, 12 Solos op. 1 . . . . . . . . . . . . . . . 5 -

оp. …............ . . $3 \ldots$

\section{Solos à Flute Travers \& Basse.}

Quanten (Cher. de) 6 Solos op. 1 . . . . . . . . . . . . ...

*Reinards. 6 Solos op. 2 . . . . . . . . . . . . . . . 3 --

Reinards 6 Solos op. 5 . . . . . . . . . . . . . . . . . $3 \ldots$

Zieleke. 6 Solos, op. 1. au mois d'May. 1778 . . . . . . . 3-10

\section{Pieces \& petits Airs pour le Clavecin.}

Abel, 6 Sonat. al l'usage de commencens . . . . . . . . . 2 ....

Bach (J. C.) 6 Sonat. op. J. . . . . . . . . . . . . . . . 4-

-_ (C. F. E.) 6 Sonat. op. 1. . . . . . . . . . . . . 4 -

*Colizzi Air Fr. variée pour le Clares. . . . . . . . . . . - -16

*...- Air Varié, vous l'ordonnes d... . . . . . . . . . . 42

*.... Marche à plusieurs Instruments. . . . . . . . . . . -12

*_. pour le Clavecin . . . . . . . . . . . . . . . .

*Entr'Acte de l'Opera Henry IV pour le Clavecin . . . . . . -19

Eichner, 6 Sonatines op. 6 . . . . . . . . . . . . . . 2-10

Neuman Clanson Français varićc . . . . . . . . . . . -14)

Neuman Overture du Deserteur . . . . . . . . . . . . . . 12

*Dirertigsement Militair . . . . . . . . . . . . . . -16

Fischer, Rondeau variée par Diet\% . . . . . . . . . . . 1 -

*Gautier, 6 Sonates op. 2. . . . . . . . . . . . . . . 3 -

Haydn, 6 Sonates op. 13 . . . . . . . . . . . . . . . 4 .

- 6 grand son. op. 14. au mois de Juillet. 1778 . . . . . 4 -. 
Howsuer, 6 Sonales, op. 1 . . . . . . . . . . . . . . 4

-6 Sonates op. 2 . . . . . . . . . . . . . . . . 4

Johnsen 6 Fugue. . . . . . . . . . . . . . . . . . . . . . . . 9

Jozzi, 8 Sonates. op. 1. . . . . . . . . . . . . . . . 4-

"Just, 6 Sonat. à l'usage des Comnencens, op. 3 . . . . . . 1-16

*_-_ Marche la Garde passe, varié . . . . . . . . . . . 12

*_-_ Air Fr. Variée ponir le Clilaress. . . . . . . . . . . 12

Kirnberger, \& Fúgús . . . . . . . . . . . . . . . . 1-16

*Kelner, 6 Fugue . . . . . . . . . . . . . . . . . . 2 -..

Klöfner, 6 Sónates, op. 6. . . . . . . . . . . . . . . 3-10

Marburg, Fugue et Capricrj. . . . . . . . . . . . . . 2 -

XII. Menuet, variée. . . . . . . . . . . . . . . . . 1 .

Michelet, 6 Sonates op. 1 . . . . . . . . . . . . . . 3 3

Plecies choisles . . . . . . . . . . . . . . . . . . 15

*Recueil des Airs, March. \& Men. . . . . . . . . . . . . 16

Paganelli 6 Sonatines . . . . . . . . . . . . . . . . 1-10

Schmugel, Fuges \& prelude pour l'orgue . . . . . . . . . 2

Schröter, 6 Sonates op. 1 . . . . . . . . . . . . . . 3-10

Tranti, 6 Sonates op. 1 . . . . . . . . . . . . . . . 3

Graaf, Air varié par Mozart . . . . . . . . . . . . . ${ }_{-12}$

Wilh. van Nassau varić par le mème . . . . . . . . . . $\quad$-6

Wagenseil, 3 Divertissement. . . . . . . . . . . . . . 1-4

De Zangwyzen der CL. Psaldien Darids en der Lofgesangen, so als dezelve in de Gereformeerde Kerken dezer Landen gebruikelyk zyn, opgestelt voor zulke die de Batso Continuo niet verstaan. Door F. G. Micholet . . . . . . . . . . . 6-

\section{Airs Française, Italien, \& Holland.}

* Extrait des cirs Françaís de tous les Operás nouveaux qui ont été representés, apropriés pour le chant ou la Flnte, avec la Basse continuë, Partie 1.2. 3. 4. 5.6. 7. 8. 9. et 10. chaque partie $6-$ Partie 6. est avec des instruments séparés . . . . . . . 12 Fleurs des Airs de diverses Op. Franç. avec l'acémpag. des Viol. Part. I . . . . . . . . . . . . . . . . . . . 3- 


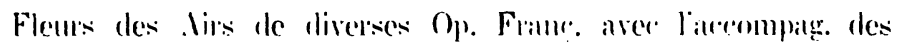

Vinl. Parl. II. . . . . . . . . . . . . . . . . . . 3 -

Duo I. . Mh! mon ami. aree lauc(ompl. . . . . . . . . . . 1-5

II. Le Piquel. aver liaromp. . . . . . . . . . . . . . 1-5

-.. III. Les voila patie aver lareromp. . . . . . . . . . . 1-j

lir I. Ah? je respire aree liaremp. . . . . . . . . . . . . 1 .

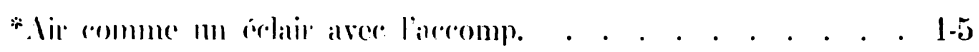

* Air Est il un sont. 1778 . . . . . . . . . . . . . . . . . . . . . .

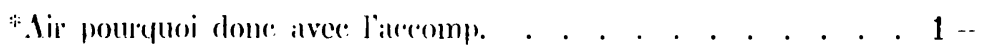

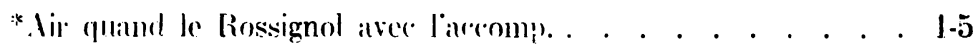

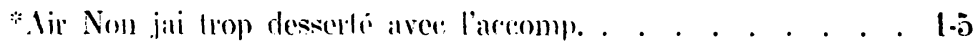

Deno les Proverhes . . . . . . . . . . . . . . . . .

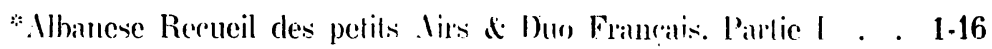

*..... Partic II . . . . . . . . . . . . . . . . . . 1-16

* _......... Partic III . . . . . . . . . . . . . . . . . . 116

* - Dun la Toillere. aver l'acento. . . . . . . . . . 1-5

* Air les Ombres Chinoises. . . . . . . . . . . . . . . . . . $\quad-6$

*-. pour le Clavecin . . . . . . . . . . . . . . . . . 12

*Dans Paris la grande Ville . . . . . . . . . . . . . . 6

Lucile, quatuor avec l'accompag. . . . . . . . . . . . . . . 2 -

Bach, Air Italien avec l'accomp. . . . . . . . . . . . . 1-10

*Colizzi, Airs Italiens . . . . . . . . . . . . . . . . 1.10

*Giordani 6 Duetti Ital. . . . . . . . . . . . . . . . 1-10

Schultz, Gans. Italien . . . . . . . . . . . . . . . . 1-10

Quelici, Cans. Italien . . . . . . . . . . . . . . . . 1-4

*Vento 6 Clans. Ital. . . . . . . . . . . . . . . . . . . . 2

De vier Musicale Jaargetyden Duitsche Zang-Airtjes, met een Basse Continuo.

Eerste Stukje verbeeldende de Zomer. . . . . . . . . 1-16

Tweede - de Herfst. . . . . . . . . . . . . . . 1-16

Derde - de Winter . . . . . . . . . . . . . . . $1-16$

Vierde - de Lente . . . . . . . . . . . . . . . 1-16

Doch het complete Werk voor . . . . . . . . . . . 6 -

Mahant, nieuwe geopende Musicale Tydkorting. . . . . . . 1-16 


\section{Diverses Airs \& Menuets pour le Violon. fl. sols}

Borlin Xll Menuel alvec Cors . . . . . . . . . . . 1-12

*Divertissem Militair, antenanl les Marches des Guardes Hol-

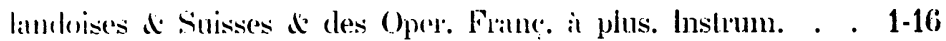

Francisconi Varche la garde palsse, variée, pour le V'iolon • • -6

Reinards la garde passe, vill. pour lil Flul. . . . . . . . . -12

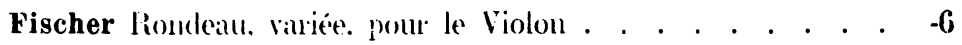

Haydn Menuet aver clors. . . . . . . . . . . . . . 1-10

Reminets 12 billo Nennels alver Cons . . . . . . . . . . 1-10

Morigi. Mentlets . . . . . . . . . . . . . . . . . . 16

Nieuwe Hollindse Dansen en Menuelten . . . . . . . . . 1-4

Verzamelinge rim Airtjes, Vauderilles, ch Contredansen . . . 1 -

\section{Diverses Traittes de Musique.}

*Boutmy 'Trailé Abrégé sur la Basse continue, dit mede in 't Duitsch 3-10

Lanzetta, principes ou l'application de Violoncello . . . . . 2 --

Mahaut nouvelle Meth. pour aprendre en peu de tems a jouer de la Fl. Trav. Fransch en Duitsch . . . . . . . . . . 1:16

Pasquali la Basse Continuë rendue aisće à liusage des commencens, avec plusieur's Exempl. Nolés, revuë d' augmenté par J. W. Lustig. Frans en Duitsch. . . . . . . . . . . . 2:10

Lustig, Inleiding tot de Musik-kunde. tweede Druk, van den Autheur ingekogt . . . . . . . . . . . . . . . . . 1 -

Marburg Aanleiding tot het Clavier speelen, vertaald door J. W.

Lustig . . . . . . . . . . . . . . . . . . . 1:16

_-_ tweede Deel, Frans en Duitsch. . . . . . . . . . . 2 -

Fritz Onderwys om Orgels en Clavieren te stemmen . . . . : 6

Les Piéces marqué en forme d'étoile* s'ont de l'impression de B. Hummel, à la Haye.

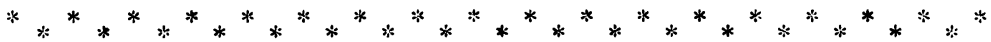

Chés les susdits Hummel on trouve de plus, tout ce qui apartient à une Boutique de Musique complette; savoir: de Papier reglé double Elephant, en diverses formats, en Main \& Rames, Relié ou en Feuille.

$I l$ vend $\&$ achête toutes sortes d'Instruments de Musique, comme: de tr'ès bons \& ordinaires Violons, Tailles de Violons, Violes de Gambe, 
Violes d'Amour, Basses, Guitarres, Harpes, Clavessins of Contıelasses; des Hautbois \& Bassons; des Flutes d' Ivoire, le Bois, d'kbene, de de Bois, tant de Grensen, que llaulres Maitres; des Flutes d'Amour. Illutes Tierces, Flutes Octaves, Flutes à Bec, Clarinuttes, Cors dle C'hassse ordinaires, \& d'une toute particulieve fa!on, dont on joïe tous les I'ons.s sur une paire, des Trompeltes, des Archets, des Cuisses de Violon, des Anches pour les Hautbois de Bassons, Boites à Resine, Cordles de Clavessin; comme aussi des meilleures C'ordes iles Violons, des Basses * Contrebasses, tout en juste prix.

N.B. Cette Catalogue ainsi que celle des Instrumenls so distribur gratis chés les susdits de lenrs Corrpspondanls. 